\title{
Changes in plasma and liver glutathione levels in Atlantic salmon Salmo salar suffering from infectious salmon anemia (ISA)
}

\author{
Brit Hjeltnes $^{1}$, Ole Bent Samuelsen ${ }^{2 * *}$ Asbjørn Martin Svardal ${ }^{2}$ \\ ' Institute of Marine Research, Division of Aquaculture, PO Box 1870, N-5024 Bergen, Norway \\ ${ }^{2}$ Department of Pharmacology and Toxicology, University of Bergen, School of Medicine, Armauer Hansens Hus, \\ N-5021 Bergen, Norway
}

\begin{abstract}
The levels of reduced glutathione (GSH) in plasma and liver in healthy Atlantic salmon Salmo salar and salmon suffering from infectious salmon anaemia (ISA) were measured using highperformance liquid chromatography (HPLC). The infected salmon had an increased level of plasma GSH compared to controls, the mean values being $72.31 \pm 11.56$ and $52.8 \pm 2.92 \mu \mathrm{M}$, respectively. The increased GSH level in plasma was found to be significant ( $p<0.0001$, unpaired $t$-test) and may be a result of intracellular release. The hepatic GSH level was significantly lower in the infected salmons compared to controls $(p<0.0001$, unpaired $t$-test $)$ and the mean values were calculated to be $1618.7 \pm 543.7$ and $2512.1 \pm 326.9 \mathrm{nmol} \mathrm{g}^{-1}$ liver (wet wt), respectively. A decrease in hepatic GSH of up to $70 \%$ in diseased fish compared to controls was observed. Such low levels of GSH in the liver may affect the capability of this organ in the transformation and excretion of xenobiotics from the body.
\end{abstract}

\section{INTRODUCTION}

Infectious salmon anemia (ISA) was first reported in Norway in 1984 in a stock of Atlantic salmon Salmo salar parr in a hatchery located on the west coast (Thorud 1991). Since then, ISA has rapidly spread along most of the Norwegian coast from the southwest region to the far north and eventually became one of the most serious diseases in Norwegian fish farming. In 1990, 140 fish farms were affected causing mortalities in the infected farms in the range of 15 to $100 \%$ (Thorud 1991). The infectious nature of the disease was demonstrated by Thorud \& Djupvik (1988) and transmission trials indicate a viral cause of the disease (Thorud 1991). The macroscopic changes in fish suffering from ISA include pale gills, dark and plethoric liver and spleen and haemorrhages in the peritoneum. The haematocrit ( $\mathrm{Hct}$ ) values are low and act as an indicator for the anaemic condition. At very low hematocrit values the histological changes in different organs are significant, the most serious being the macroscopic and histological changes in the liver.

Since the liver is commonly affected in ISA, it is

\footnotetext{
- Addressee for correspondence
}

plausible that glutathione (L- $\gamma$-glutamyl-L-cysteinyl glycine) may contribute to the pathogenesis. Animal liver contains glutathione (GSH) in the range of 0.5 to $10 \mathrm{mM}$ and the major part ( $>90 \%$ ) of the plasma GSH is produced by this organ (Meister \& Anderson 1983). Extracellular GSH protects cells of diverse tissues against oxidation injury and is the major transport form of the amino acid cysteine. Within cells, GSH is the dominant intracellular thiol and has an important function in the cellular defence against oxidative injury (Meister \& Anderson 1983, Meister 1988). Conjugation with GSH is an important detoxication reaction for electrophilic xenobiotics. Such electrophiles are most often formed in the body by metabolizing enzymes and may be responsible for a number of adverse effects (Meister \& Anderson 1983, Meister 1988). GSH is also believed to be important in the initiation and progression of lymphocyte activation, and thus essential for host defence. Specifically, depletion of intracellular GSH inhibits lymphocyte activation by mitogens, and GSH is critical for the function of natural killer cells and for lymphocyte-mediated cytotoxicity (Fischman et al. 1981, Hamilos \& Wedner 1985, Wedner et al. 1985 Droege et al. 1986, MacDermott et al. 1986, Buhl et al 1989, Roederer et al. 1990, Suthanthiran et al. 1990). To 
assess whether ISA may be associated with deficiency of hepatic and extracellular GSH, the concentration of this substance were measured both in liver and plasma.

\section{MATERIALS AND METHODS}

Heparinized blood was collected from Atlantic salmon at a fish farm (Sotra, Norway) during a natural outbreak of ISA. The blood cells were washed 3 times in EMEM (Earle's modification of Minimum Essential Medium), sonicated for $10 \mathrm{~s}$, and then reconstituted into their original volume in EMEM. The experimental fish were challenged by an intraperitoneal injection of $0.2 \mathrm{ml}$ of the blood cell homogenate diluted 1:10 in EMEM. For injection of the 10 control fish, blood from apparently healthy fish from the stock held at the Institute of Marine Research (Bergen, Norway) was employed. The fish used in this experiment were Atlantic salmon smolts weighing approximately $100 \mathrm{~g}$. They were kept in UV-treated water with a salinity of $0.3 \%$ in aquaria (200 l) at a temperature of 10 to $12{ }^{\circ} \mathrm{C}$. During the experiment, the fish were fed commercially available dry pellets (Ewos, Norway).

When a fish showed signs of being ill, it was anaesthetized and blood samples were collected in heparinized syringes. The blood was centrifuged at $14000 \times g$ and the plasma retained. The plasma was immediately frozen in liquid nitrogen and kept at $-80^{\circ} \mathrm{C}$ until analysed. The plasma samples were processed and analysed for GSH following the method of Svardal et al. (1990).

Immediately after killing the fish, liver samples were frozen in liquid nitrogen, brought to the laboratory and kept at $-80^{\circ} \mathrm{C}$ until analysed. The samples were processed according to the procedure described by Djuurhuus et al. (1988) and analysed for GSH following the method of Svardal et al. (1990). Samples of the control fish were taken $4 \mathrm{wk}$ following the intraperitoneal injection of blood homogenate. Data obtained were statistically analyzed using unpaired $t$-tests.

\section{RESULTS}

After 3 wk the infected fish stopped feeding, darkened, became lethargic and started to die. Dead and moribund fish exhibited haemorrhages on the eyes, ascites, had pale gills and heart, petechiation on the visceral fat and enlargement of the spleen and liver. The mean haematocrit (Hct) value of the infected fish $(17.22 \% \pm 9.16)$ were significantly lower $(p<0.0001$, unpaired $t$-test) compared to the control fish $(37.11 \%$ \pm 3.74). Histopathological inspection of the livers revealed extensive haemorrhages and zonal necrosis. The results of the haematocrit and GSH analysis of liver and plasma is shown in Fig. 1.

Most of the infected salmons had an increased level of plasma GSH compared to control fish. The mean values were calculated to be $72.31 \pm 11.56$ and $52.8 \pm$ $2.92 \mu \mathrm{M}$ for the infected and the control fish, respectively, giving a significant increase $(\mathrm{p}<0.0001$, unpaired $t$-test). Problems with blood sampling of the infected fish was the reason that only 12 plasma samples were available for analysis.

The hepatic GSH level, however, was significantly lower in the infected salmons compared to controls $(\mathrm{p}<0.0001$, unpaired $t$-test $)$ and the mean values were calculated to be $1618.7 \pm 543.7$ and $2512.1 \pm 326.9$ nmol $\mathrm{g}^{-1}$ liver (wet $\mathrm{wt}$ ), respectively. A decrease in hepatic GSH of up to $70 \%$ in 16 out of 20 diseased fish compared to controls was observed

All the infected fish from which samples were taken displayed symptoms.
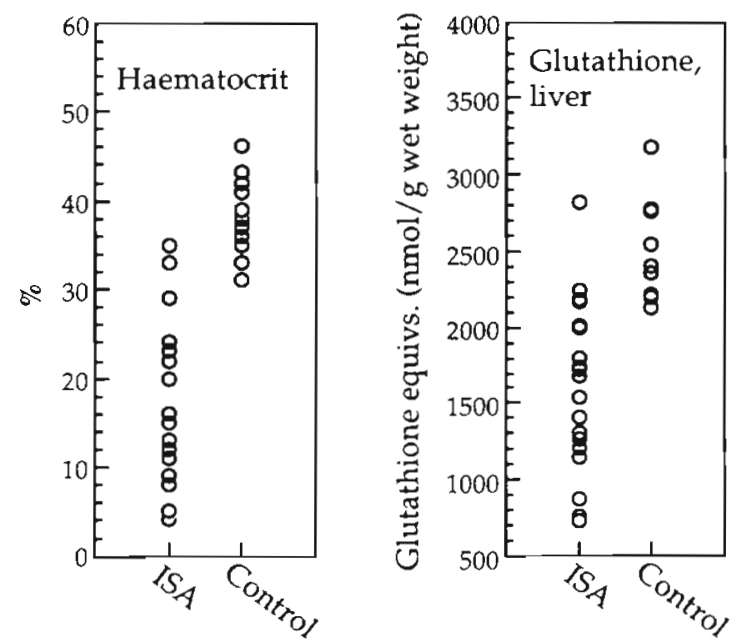

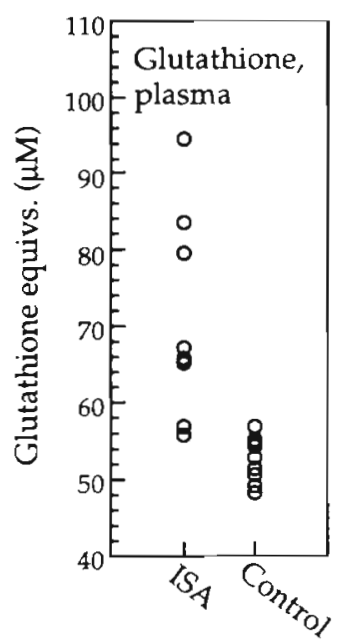

Fig. 1. Salmo salar. Haematocrit, plasma and liver content of reduced glutathione (GSH) in control fish and fish suffering from Infectious Salmon Anemia (ISA) 


\section{DISCUSSION}

All of the physiological abnormalities described are typical findings from ISA infected fish (Thorud \& Djupvik 1988, Evensen et al. 1991) and there can be no doubt that the fish were in fact suffering from this disease. GSH is synthesized in the liver and this organ is the main depot for this substance. The plasma level of GSH is a balance between secretion from the liver and elimination through the kidneys. Of 20 salmon suffering from ISA 16 had a lowered level of GSH in the liver. Hence, decreased levels of plasma GSH should have occurred. However, plasma levels of GSH in most of the infected fish were higher than in the control fish. Examination of blood from ISA diseased fish has shown the presence of lysed empty red blood cells (ghost cells). Since the concentration of GSH in extracellular media like plasma is orders of magnitude lower than the intracellular content, the elevated GSH level in plasma may be due to leakage from these lysed cells.

It has been proposed that GSH deficiency in plasma may have a negative effect on the immune function. Whether the increased GSH level in plasma of ISA infected fish brings about an increase in the GSH content in for example lymphocytes and thus possibly stimulates the immune system or if, the sickness per se results in a decline in the GSH levels in these cells, as demonstrated for the liver, remains to be determined.

Due to significant lower levels of GSH in the liver of ISA infected fish compared to control fish, the liver in the diseased fish may be less capable in the transformation and excretion of xenobiotics from the body and, consequently, a reduction in detoxifying capacity of the liver may lead to enhanced risk of xenobiotic damage of this organ.

Acknowledgements. We thank Mr H. Rudra and Mr A. Hoylandskjær for valuable help during sampling and Prof. R. Scheline for critical comments on the manuscript.

\section{LITERATURE CITED}

Buhl, R., Holroyd, K. J., Mastrangeli, A., Cantin, A. M., Jaffe, H. A., Wells, F. B., Saltini, C., Crystal, R. G. (1989). Systemic glutathione deficiency in symptom-free HIVseropositive individuals. Lancet 1989(2): 1294-1298

Djurhuus, R., Svardal, A. M., Ueland, P. M., Male, R., Lil-

Responsible Subject Editor: O. Kinne, Oldendorf/Luhe, Germany lehaug, J. R. (1988). Growth support and toxicity of homocystein and its effects on methionine in non-transformed and chemically transformed $\mathrm{C} 3 \mathrm{H} / 10 \mathrm{TI} / 2$ cells. Carcinogenesis 9: 9-16

Droege, W., Pottmeyer-Gerber, C., Schmidt, H., Nick, S. (1986). Glutathione augments the activation of cytotoxic $T$ lymphocytes in vivo. Immunobiology 172: 151-156

Evensen, Ø., Thorud, K. E., Olsen, Y. A. (1991). A morphological study of the gross and light microscopic lessions of infectious anaemia in Atlantic salmon (Salmo salar). Res. Vet. Sci. 51: 215-222

Fischman, C. M., Udey, M. C., Kurtz, M., Wedner, H. J. (1981). Inhibition of lectin induced lymphocyte activation by 2 . cyclohexene-1-one: decreased intracellular glutathione inhibits an early event in the activation sequence. $J$ Immunol. 127: 2257-2262.

Hamilos, D. L., Wedner, H. J. (1985). The role of glutathione in lymphocyte activation. I. Comparison of inhibitory effects of buthionine sulfoximidine and 2cyclohexene-1-one by nuclear size transformation. J. Immunol. 135: 2740-2747

MacDermott, R. P., Bertovich, M. J., Bragdon, M. J., Nash, G S., Leusch, M. S., Wedner, H. J. (1986). Inhibition of cellmediated cytotoxicity by 2-cyclohexene-1-one: evidence for a role for glutathione and/or glutathione-protein interactions in cytolysis. Immunology 57: 521-526

Meister, A. (1988). Glutathione metabolism and its selective modification. J. biol. Chem. 263: 17205-17208

Meister, A., Anderson, M. E. (1983). Glutathione. A. Rev Biochem. 52: 711-760

Roederer, M., Staal, F. J. T., Raju, P. A., Ela, S. W., Herzenberg, L. A. (1990). Cytokine-stimulated human immunodeficiency virus replication is inhibited by $N$ acetyl-L-cysteine. Proc. natl. Acad. Sci. U.S.A. 87 $4884-4888$

Suthanthiran, M., Andersen, M. E., Sharma, V. K., Meister, A (1990). Glutathione regulates activation-dependant DNA synthesis in highly purified normal human T lymphocytes stimulated via the CD2 and CD3 antigens. Proc. natl. Acad Sci. U.S.A. 87 : $3343-3347$

Svardal, A. M., Mansoor, M. A., Ueland, P. M. (1990). Determination of reduced, oxidised and protein-bound glutathione in human plasma with precolumn derivatization with monobromobimane and liquid chromatography. Analyt. Biochem. 184: 338-346

Thorud, K. (1991). Infectious salmon anaemia. Transmission trials. Haematological, clinical chemical and morphological investigations. Dr Scient thesis, Norwegian College of Vetrinary Medicine

Thorud, K., Djupvik, H. O. (1988). Infectious anaemia in Atlantic salmon (Salmo salar L.). Bull. Eur. Ass. fish Pathol. 8: $109-111$

Wedner, H. J., Bahn, G., Gordon, L. K., Fischman, C. M. (1985). Inhibition of lectin-induced lymphocyte activation by 2-cyclohexene-1-one: analysis of DNA synthesis in individual cells by BUdR quenching of Hoechst 33258. Int. J. Immunopharmacol. 7: 25-30

Manuscript first received: January 2, 1992

Revised version accepted: June 30, 1992 